Tropical Journal of Pharmaceutical Research July 2018; 17 (7): 1445-1451

ISSN: $1596-5996$ (print); 1596-9827 (electronic)

(C) Pharmacotherapy Group, Faculty of Pharmacy, University of Benin, Benin City, 300001 Nigeria.

\title{
Application of statins in management of glioma: Recent advances
}

\author{
Qinyi $\mathrm{Xu}^{1}$, Jun Liu ${ }^{1}$, Dong Wang ${ }^{1}$, Yinong $\mathrm{Xu}^{3}$, Meng $\mathrm{Ji}^{3}$, Junhui Chen ${ }^{2 \star}$, Yuhai \\ Wang $^{2}$ \\ ${ }^{1}$ Department of Neurosurgery, Huishan People's Hospital, ${ }^{2}$ Department of Neurosurgery, I0I Hospital of PLA, Wuxi 214044, \\ ${ }^{3}$ Department of Neurosurgery, Taizhou No.4 Hospital, Taizhou 225300, China
}

*For correspondence: Email: chenjunhui1110@126.com

Sent for review: 22 February 2018

Revised accepted: 21 June 2018

\begin{abstract}
Gliomas are common primary intra-cerebral tumors in adults, and seriously threaten the health and life of affected patients, especially highly-malignant gliomas, such as glioblastoma multiforme. The clinical prognosis of glioma patients is poor, even for those who have received comprehensive treatment including surgery and concurrent chemo- and/or radio-therapy. As a structural analog of $\beta$-hydroxy- $\beta$ methylglutaryl coenzyme $A$ (HMG CoA) reductase, statins are a restrictive enzyme in the metabolism of cholesterol. Recent laboratory studies and clinical trials have demonstrated that statins can exert antitumor effect, improve clinical prognosis and significantly prolong the survival time of glioma patients. This article is aimed to highlight the mechanisms of the anti-glioma effect of statins and review recent advances in the management of the disease.
\end{abstract}

Keywords: Glioma, Glioblastoma multiforme, Intra-cerebral tumors, Statins, Prognosis, Survival time, $\beta$-Hydroxy- $\beta$-methylglutaryl coenzyme A (HMG CoA) reductase

\footnotetext{
This is an Open Access article that uses a funding model which does not charge readers or their institutions for access and distributed under the terms of the Creative Commons Attribution License (http://creativecommons.org/licenses/by/4.0) and the Budapest Open Access Initiative (http://www.budapestopenaccessinitiative.org/read), which permit unrestricted use, distribution, and reproduction in any medium, provided the original work is properly credited.

Tropical Journal of Pharmaceutical Research is indexed by Science Citation Index (SciSearch), Scopus, International Pharmaceutical Abstract, Chemical Abstracts, Embase, Index Copernicus, EBSCO, African Index Medicus, JournalSeek, Journal Citation Reports/Science Edition, Directory of Open Access Journals (DOAJ), African Journal Online, Bioline International, Open-J-Gate and Pharmacy Abstracts
}

\section{INTRODUCTION}

As a type of intra-cerebral neoplasm, gliomas are derived from the glial cells of the brain or the spine. According to the World Health Organization (WHO), gliomas can be classified into four malignancy grades I-IV [1-3]. Malignant gliomas are classified as a type of grade IV tumor, which are the most aggressive and highlyinvasive tumors of the central nervous system with an annual incidence rate of approximately $0.03-0.05 \%$ worldwide [4,5]. As the most common type of malignant glioma, glioblastoma multiforme (GBM) is another category of glioma, accounting for $30 \%$ of primary brain tumors [6]. Despite recent advancements in the treatment of GBM, the clinical prognosis of GBM patients remains poor. Following the standard-of-care treatment, such as surgical resection and chemoor radio-therapy, GBM patients obtain a median survival of 15 months. The 5-year survival rate merely achieves $3.3 \%[7,8]$.

Statins are common prescription drugs to lower the serum level of lipid, treat dyslipidemias and prevent cardiovascular diseases [9]. In addition, statins have been proven to exert multiple biological functions including anti-inflammation 
[10], anti-oxidant [11,12], angiogenic and vascular protection $[13,14]$, and anti-cancer effects [15-19]. Previous studies have indicated the potential chemo-preventive role of statins upon the risk of malignant tumors and statins can reduce the mortality rate of cancer patients and the incidence rate of gliomas [15-22]. Previous laboratory studies have demonstrated that statins can exert anti-tumor effect through inhibiting the growth, cellular proliferation and migration by inducing cellular apoptosis [15,18,23-26]. In this article, we summarized the most recent studies related to the role of statins in gliomas, aiming to unravel the potential mechanism underlying the anti-glioma effect of statins.

\section{EFFECT OF STATINS ON GLIOMA CELL PROLIFERATION}

As a type of primary intra-cerebral tumor, the clinical prognosis of patients diagnosed with gliomas is still poor mainly due to the alarmingly high proliferation rate of glioma cells. How to suppress and prevent the rapid proliferation of glioma cells has captivated widespread attentions from the oncologists. Previous studies have demonstrated that statins are able to suppress the invasion and growth of HMG-CoA$\mathrm{R}$ and glioma cells and function to induce the cellular apoptosis $[28,29]$. Recent studies have indicated that the incidence of glioma is considerably decreased in those who are administered with long-term statins. Moreover, statins can promote the apoptosis of glioma cells and suppress the proliferation of tumor cells [18,25-27].

\section{Fas translocation and PI3K/Akt/mTOR, caspase-3}

$\mathrm{PI3K} /$ Akt signaling pathway is involved in the proliferation, migration and invasion of glioma cells [30]. Wu et al [25] have proposed that simvastatin can suppress the cellular proliferation and migration, and induce the apoptosis of U87 and U251 cells in a dose- and time-dependent pattern. Simvastatin is capable of lowering the cholesterol level on the glioma cell membrane, disrupting the structure of lipid rafts and downregulating the PI3K/Akt pathway. Additionally, the signaling mechanism of Fas translocation and $\mathrm{PI3K} /$ Akt/caspase-3 pathway is involved with the anti-tumor effects of simvastatin. Maja Misirkica et al [31] have also demonstrated that simvastatin can mediate the induction of Beclin-1 and autophagy of glioma cells are correlated with the down-regulation of basal mTOR activity.

Simvastatin has been proven to trigger the AMPK/Akt/mTOR autophagy, prevent glioma cells from apoptotic death. In addition, statins have been validated to induce cell autophagy and regulate cell necrosis [32-35]. Therefore, Akt-related molecular signaling pathways are of vital significance in the proliferation of glioma cells. HMG CoA reductase specifically suppresses the expression of Akt by inhibiting AMPK or PI3K activity, thereby suppressing the cell autophagy pathway and promoting the necrosis of glioma cells, as illustrated in Figure 1.

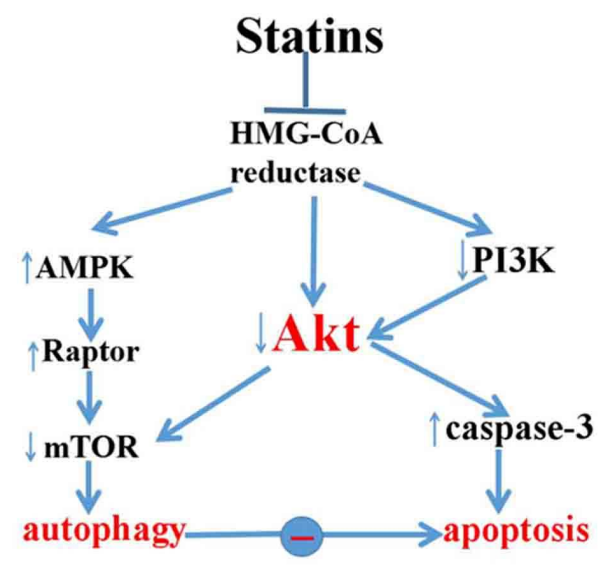

Figure 1: Molecular signaling pathways of autophagy and apoptosis by Akt

\section{JNK/ATF-2 signaling pathway}

JNK/ATF-2 molecular signaling pathway is intimately correlated with cell proliferation. The trans-activation capacity of $\mathrm{N}$-terminal domains of $\mathrm{c}$-jun and ATF-2 is strengthened via the phosphorylation of JNK and p38 kinase [36]. ATF-2 is involved in JNK- and p38 kinasedependent ATF-2 N-terminal phosphorylation to cell stress, which has been validated in multiple cellular stress responses [37]. Meral Koyuturk et al [38] have also demonstrated that simvastatin can reduce cell proliferation and induce apoptotic death in C6 glioma cells in a time- and concentration-dependent pattern. The mechanism is the simvastatin-induced activation of JNK in glioma cells via the induction of $c$-jun and the activation of ATF-2. Subsequently, the death of glioma cells is aggravated. Ohba et al [39] have found that similar molecular mechanisms of c-Jun-associated responses are vital events in the cyto-protection of temozolomide (TMZ)-treated glioma cells mediated by JNK.

\section{TRAIL signaling pathway}

The tumor necrosis factor-related apoptosisinducing ligand (TRAIL) is a member of the TNF super-family, and able to bind to death receptors including DR5 and DR4, thereby inducing cell apoptosis in multiple cancer cells but inducing no 
harm to normal cells [40-42]. David et al [42] have demonstrated that lovastatin can sensitize the TRAIL-induced cell death, and two of the tested glioblastoma cell lines are resistant to TRAIL-induced apoptosis. Therefore, lovastatin can sensitize glioblastoma cells via the effect upon the TRAIL receptor signaling pathway, and equally triggers an unknown mechanism.

Lovastatin is a cytostatic agent that supports TRAIL-induced cell apoptosis in glioblastoma cells. TRAIL-induced cell death mechanism has been evaluated in the colon and pulmonary tumors [43]. Combined regime of TRAIL, nonchemotherapeutic agents and statins might provide a potentially novel option for the clinical treatment of gliomas.

\section{Raf/MEK/ERK signaling pathway}

Changes in the Ras/Rho-mediated signaling transduction, including the Ras-Raf-MEK-ERK signaling pathway, play a central role in the molecular pathogenesis of GBM $[5,18]$. Sarah et al [18] have reported that lovastatin and perillyl alcohol disrupt the regulatory effect of Ras-RafMEK-ERK and MVA- and the signaling pathways in U343 and U87 cells, which equally affect the modification of $\mathrm{H}$-Ras and Rac1 post-translation, and data on these compounds strengthen the effect of isoprenoids FPP and GGPP upon modification of small GTPases post-translation and accelerating the proliferation of glioma cells. Statins plays an essential role in alternative types of cancer.

Wu et al [44] have found that statins are able to trigger cell apoptosis by regulating the Raf/MEK/ERK signaling pathway. Downregulating the Raf/MEK/ERK signaling pathway can potentiate cell apoptosis induced by statins when exposed to D98059, a MEK1 inhibitor and can sensitize AML cells to a low concentration of lovastatin. Du et al [45] have demonstrated that cell apoptosis induced by dihydroartemisinin is accompanied with the PI3K/AKT and Raf/MEK/ERK signaling pathway inactivation, besides down-regulating the expression of $\mathrm{Mcl}-1$ and Bcl-2, anti-apoptotic proteins. Roberts et al [46] have demonstrated that dys-regulation of the Raf/MEK/ERK signaling pathway is correlated with the incidence of multiple types of human tumors and suppressing the Raf/MEK/ERK signaling pathway serves as a novel option for cancer management.

\section{Alternative signaling pathways}

Previous studies have proposed multiple mechanisms and molecular signaling pathways are associated with the effect of statins upon inducing the apoptosis of glioma cells. However, which signaling pathway plays the most pivotal role in these events have been largely unknown. Accumulated recent evidence has demonstrated that the cell autophagy-related signaling pathways, such as the $\mathrm{PI} / 3 \mathrm{~K} / \mathrm{Akt} / \mathrm{mTOR}$ and MAPK signaling pathways, exert the primary effect upon the death and apoptosis of glioma cells $[32-35,47]$.

\section{EFFECT OF STATINS ON GLIOMA CELL INVASION}

Cancer invasion is a very important indicator in terms of evaluating the cancer malignancy. Recent studies have shown that microglia can promote the growth of glioma cells [48]. Glioma cells can activate the microglia to express matrix metalloprotease (MMPs), which can promote the glioma cell invasion. Some studies have also demonstrated that atorvastatin can downregulate the expression levels of matrix metalloprotease 2 (MMP-2), MMP-14 and MMP9 , whereas it suppresses the invasion of osteosarcoma by suppressing MMP-2 activity via RhoA-JNK-c-Jun-MMP2 signaling pathway [4952].

Yi et al [53] have demonstrated that atorvastatin can mitigate the pro-tumorigenic effect of microglia upon the glioma invasion and migration by down-regulating MT1-MMP expression in microglia. A potential mechanism of downregulating MT1-MMP in microglia is associated with the P38 MAPK signaling pathway. Fromigue et al [52] also reported that inhibiting HMG-CoA reductase via statins and decreasing RhoAGTPase prenylation reduces MMP2 activity in the JNK-cJun signaling pathway of osteosarcoma cells. Markovic et al [48] have found that glioma manipulates tumor-associated microglial cells through TLR signaling pathway for MT1-MMP expression, thereby promoting the expansion of glioma. The pro-tumorigenic effect of microglial cells serves as a target for the management of new brain tumors.

Statins significantly down-regulate MMP-2 expression, which can reduce the invasion of glioma cells. It also acts as a promising tool to reduce the invasion of microglia-promoted tumors. Regarding other mechanisms, Cordle et al [54] have shown that statins can inhibit the actions of Rho GTPases of microglia. Sundararaj et al [55] have demonstrated that inhibiting LPSindiced ERK activation by simvastatin is counteracted by GGPP and simvastatin can suppress MMP-1 expression induced-LPS in U937 cells via targeting ERK activation mediated 
by protein isoprenylation. These findings indicate that statins can suppress the invasion of glioma cells and enhance the clinical prognosis of glioma patients.

\section{EFFECT OF STATINS ON GLIOMA CELL MIGRATION}

Although distant migration of glioma is rare, it also indicates the end-stage of the disease and a poor outcome. Glioma cell migration interferes with a good prognosis. Statins may play an important role in preventing glioma migration $[15,18,20,26,31]$. However, the specific molecular mechanism remains elusive. Nawaz et al [56] have indicated Cbx7 potentiation within glioma cells leads to YAP/TAZ loss, which drives the transcriptome, as evidenced by the negative enrichment of the YAP/TAZ targets. Cbx7 can regulate SAPK/JNK activity via modulating CTGF, and this Hippo signaling pathway is evaluated by detecting YAP/TAZ-dependent transcriptional activity, which is less effective in the of migration of glioblastoma.

Yi et al [53] have demonstrated atorvastatin reduces the pro-tumorigenic effect of microglia upon the migration of glioma by down-regulating the expression level of MT1-MMP. Therefore, down-regulating MT1-MMP is regulated by p38 MAPK signaling pathway in microglia. Obara et al [57] have demonstrated that cerivastatin is capable of mediating FAK tyrosine phosphorylation and influencing different cell effects by interacting with several cell signaling pathways associated with cell migration. These studies support further research on statins, as a candidate, and targeting the signal transduction pathways related to glioblastoma biology probably serves as a potential treatment for glioma. Large sample size clinical trials of statins or drugs with similar structures are urgently required to enhance the quality of life of glioma patients.

\section{EFFECT OF STATINS ON GLIOMA ANGIOGENESIS}

Angiogenesis plays a vital role in the progression and metastasis of tumors because cancer cells can retain persistent proliferation via acquiring oxygen and nutrients from the blood vessels $[58,59]$. Consequently, anti-angiogenesis is a therapeutic strategy to target malignant tumors. Inhibition of the activity of vascular endothelial growth factor (VEGF) is an effective approach to inhibit the proliferation of GBM cells.

The significance of VEGF/VEGFR-2 signaling pathway has been proven in the endothelial cell function of malignant tumors. VEGF and VEGF receptor (VEGFR) play a vital role in the lymphangiogenesis angiogenesis [60]. Recent research has demonstrated that angiogenesis is a complicated event, which regulated by multiple stimulating and inhibiting factors. Although multiple molecules associated with angiogenesis, VEGF/VEGFR signaling pathway is a major regulator of angiogenesis, and it is significantly expressed in glioblastoma and other types of cancer $[61,62]$.

Additionally, there are many possible molecular targets for modified anti-angiogenesis intervention, such as the TGF $\beta$ signaling pathway $[63,64]$, VEGFR-2/Flk-1 $[65,66]$ and the RTK/PI3K/Akt/mTOR signaling pathways [67,68], as illustrated in Figure 2 [69]. Dong Huang et al [70] have demonstrated that statins can promote the angiogenesis in diabetic rat models with $\mathrm{Ml}$, probably mediated by up-regulating the VEGFdependent Akt/eNOS pathway. Chang et al [71] have found that rosuvastatin can alleviate experimental HPS via blocking pulmonary inflammatory angiogenesis via TNF- $\alpha / N F-K B$ and down-regulation of VEGF/Rho-associated A kinase signaling pathway. Wu et al [72] have also demonstrated that simvastatin can promote TBIinduced angiogenesis at the margins of the lesions and hippocampus as well as accelerate function recovery whereas accelerate in vitro angiogenesis, which are probably associated with activation of the VEGFR-2/Akt/endothelial nitric oxide synthase signaling pathway induced by simvastatin. Both studies have demonstrated that statins play a crucial role in angiogenesistarget therapy in glioma patients. Nevertheless, a large clinical randomized controlled trial (RCT) and evidence-based medicine are still urgently needed to confirm these findings.

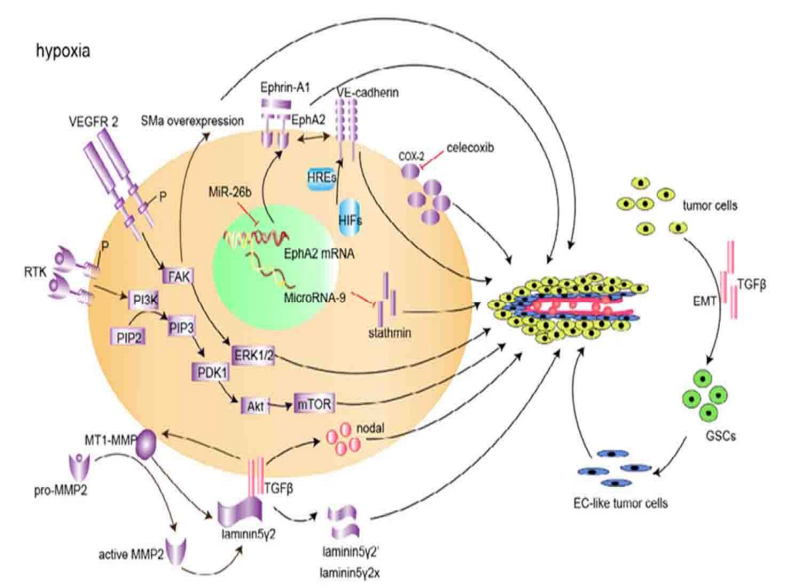

Figure 2: Schematic graph of signaling pathways involved with glioma vasculogenic mimicry (VM). VM process proceeds under hypoxic condition. Signaling molecules modulated by small inhibitory RNAs, small 
molecular inhibitors or blocking antibodies are illustrated to represent the direct or indirect effect upon VM. They are classified into embryonic/stem cell (red), microenvironment (purple) and hypoxia signaling pathways (blue). No boundary lines exist among three parts and the overlap among major VM signaling pathways indicates the coordination of these pathways

\section{CLINICAL TRIAL PROGRESS}

Many studies have confirmed that statins safe are and effective for treating glioma and other types of cancers. However, the results of clinical studies have been inconsistent and contrasting. Besides, the clinical efficacy of statins in patients with glioma has been controversial (Table 1). Unfortunately, relevant data obtained from RCT are still lacking. A nationwide case-control study including 2656 patients and 18480 healthy controls in Denmark proposed that the incidence of glioma is decreased among those with longterm use of statin their counterparts never use of statins, which is negatively associated with the intensity of statin administration [17].

Another similar clinical trial including 339 GBM patients has demonstrated that statin use before diagnosis can decrease the HR of death. HR decreases over prolonged intensity and duration of pre-diagnostic statin use [19]. Therefore, longterm pre-diagnostic statin use may improve the survival of GBM patients. In contrast, Seliger et al [73] opposed of the notion that negative association exists between use of statins and risk of glioma in a case-control investigation from the UK-based Clinical Practice Research Datalink to analyze of the administration of statins among 2469 glioma patients and 24,690 healthy controls. Ferris et al [22] have found that both statins and non-steroidal anti-inflammatory drugs possess significantly inverse trends between the duration of drug use and glioma risks, and drug intake for longer than 120 months demonstrates the most significant associations for both medication types. Another preoperative statin use study included 78 patients taking statins preoperatively, while 206 patients did not. The results had similar progression-free survival before and after propensity score matching. Also, the mortality is similar between the two groups [74]. These studies were not RCTs.

Therefore, the evidence rank is relatively low. A large sample size, randomized and placebocontrolled trial is urgently needed. Of note, there are two clinical trials about statins and glioma registered on clinicaltrials.gov. Altwairgi [75] aims to evaluate the clinical efficacy and safety of atorvastatin in combination with radiotherapy and temozolomide in glioblastoma (ClinicalTrials.gov Identifier: NCT02029573), and Centre Oscar Lambret wanted to explore safety of FluvastatinCelebrex Association in Low-grade and High Grade Optico-chiasmatic Gliomas (FLUVABREX) (ClinicalTrials.gov Identifier:NCT02115074). Further results and more RCT studies are urgent required to explore the role of statins in glioma.

\section{CONCLUDING REMARKS}

Previous studies have suggested that statins can reduce the morbidity of glioma patients with longterm use and improve the clinical prognosis and prolong the survival time of glioma patients, which has been validated by numerous laboratory studies. In addition, clinical studies have yielded negative results. Nevertheless, these negative outcomes do not interfere with further evaluation of the safety and clinical efficacy of statins administration in glioma patients. Large-scale research and RCT studies are still urgently required to investigate the clinical benefits of statin use in glioma patients.

\section{DECLARATIONS}

\section{Conflict of Interest}

No conflict of interest associated with this work.

\section{Contribution of Authors}

The authors declare that this work was done by the authors named in this article and all liabilities pertaining to claims relating to the content of this article will be borne by them.

Table 1: Summary of relevant clinical trials of statins and glioma in the period 2012 - 2016

\begin{tabular}{lllll}
\hline Clinical trial & RCT & N (s/c) & Evaluation & Conclusion \\
\hline Gaist et al [17], 2013 & No & $2656 / 18480$ & Glioma morbidity & Reduce morbidity \\
Gaist et al [19], 2014 & No & $113 / 226$ & Survival & Prolong survival. No effect \\
Seliger et al [75], 2016 & No & $2469 / 24690$ & Glioma morbidity & Reduce morbidity \\
Ferris et al [23], 2012 & No & $517 / 400$ & Glioma morbidity & No effect \\
Bhavsar et al [76], 2016 & No & $78 / 206$ & Survival and mortality & \\
\hline
\end{tabular}

Abbreviation: RCT: Randomized controlled trial, N: included patients, s: statins used and c: control group 


\section{REFERENCES}

1. DeAngelis LM Brain tumors. N Engl J Med 2001; 344: 114-123.

2. Deorah S, Lynch CF, Sibenaller ZA, Ryken TC. Trends in brain cancer incidence and survival in the United States: Surveillance, epidemiology, and end results program, 1973 to 2001. Neurosurg Focus 2006; 20: E1.

3. Louis DN, Ohgaki $H$, Wiestler OD, Cavenee WK, Burger $P C$, Jouvet A, Scheithauer BW, Kleihues $P$. The 2007 WHO classification of tumours of the central nervous system. Acta Neuropathol 2007; 114: 97-109.

4. Ostrom $Q T$, Gittleman $H$, Liao $P$, Rouse $C$, Chen $Y$, Dowling J, Wolinsky $Y$, Kruchko C, Barnholtz-Sloan J. CBTRUS statistical report: primary brain and central nervous system tumors diagnosed in the United States in 2007-2011. Neuro Oncol 2014; 16: iv1-63.

5. Lo H. Targeting Ras-RAF-ERK and its interactive pathways as a novel therapy for malignant gliomas. Curr Cancer Drug Targets 2010; 10: 840-8.

6. Dobes M, Khurana VG, Shadbolt B, Jain S, Smith SF, Smee $R$, Dexter $M$, Cook $R$. Increasing incidence of glioblastoma multiforme and meningioma, and decreasing incidence of Schwannoma (2000-2008): findings of a multicenter Australian study. Surg Neurol Int 2011; 2:176.

7. Stupp R, Hegi ME, Mason WP, van den Bent MJ, Taphoorn MJ, Janzer RC, Ludwin SK, Allgeier A, Fisher $B$, Belanger $K$, et al. Effects of radiotherapy with concomitant and adjuvant temozolomide versus radiotherapy alone on survival in glioblastoma in a randomised phase III study: 5-year analysis of the EORTC-NCIC trial. Lancet Oncol 2009; 10: 459-466

8. Bondy ML, Scheurer ME, Malmer B, Barnholtz-Sloan JS, Davis FG, ll'yasova D, Kruchko C, McCarthy BJ, Rajaraman $P$, Schwartzbaum JA, et al. Brain tumor epidemiology: consensus from the Brain Tumor Epidemiology Consortium. Cancer 2008; 113: 19531968.

9. Fletcher $B$, Berra $K$, Ades $P$, Braun $L T$, Burke $L E$, Durstine JL, Fair JM, Fletcher GF, Goff D, Hayman LL, et al. Managing abnormal blood lipids: a collaborative approach. Circulation 2005; 112: 3184-3209.

10. Sehba FA, Pluta RM, Zhang JH. Metamorphosis of subarachnoid hemorrhage research: from delayed vasospasm to early brain injury. Mol Neurobiol 2011; 43: 27-40.

11. Buyukhatlpoglu H, Sezen Y, Yildiz A, Guntekin U, Bas M, Polat M, Demirbag R, Taskin A, Celik $H$, Aksoy $N$. Effects of statin use on total oxidant and antioxidant capacity and ceruloplasmin activity. Clin Invest Med 2010; 33: E313-E320.

12. Caner B, Hou J, Altay O, Fujii M, Zhang JH. Transition of research focus from vasospasm to early brain injury after subarachnoid hemorrhage. J Neurochem 2012; 123: $12-21$

13. Budohoski KP, Czosnyka M, Kirkpatrick PJ, Smielewski $P$, Steiner LA, Pickard JD. Clinical relevance of cerebral autoregulation following subarachnoid haemorrhage. Nat Rev Neurol 2013; 9: 152-163.

14. Naraoka M, Munakata A, Matsuda N, Shimamura $N$, Ohkuma $H$. Suppression of the Rho/Rho-Kinase pathway and prevention of cerebral vasospasm by combination treatment with statin and fasudil after subarachnoid hemorrhage in rabbit. Trans/ Stroke Res 2013; 4: 368-374.

15. Gliemroth J, Zulewski H, Arnold H, Terzis AJ. Migration, proliferation, and invasion of human glioma cells following treatment with simvastatin. Neurosurg Rev 2003; 26: 117-124.

16. Murakami M, Goto T, Saito Y, Goto S, Kochi M, Ushio Y. The inhibitory effect of simvastatin on growth in malignant gliomas-with special reference to its local application with fibrin glue spray in vivo. Int $J$ Oncol 2001; 19: 525-531.

17. Gaist D, Andersen L, Hallas J, Sorensen HT, Schroder $H D$, Friis $S$. Use of statins and risk of glioma: a nationwide case-control study in Denmark. Br J Cancer 2013; 108: 715-720.

18. Afshordel S, Kern B, Clasohm J, Konig H, Priester M, Weissenberger J, Kögel D, Eckert GP Lovastatin and perillyl alcohol inhibit glioma cell invasion, migration, and proliferation-impact of Ras-/Rhoprenylation. Pharmacol Res 2015; 91: 69-77.

19. Gaist D, Hallas J, Friis $S$, Hansen S, Sørensen HT. Statin use and survival following glioblastoma multiforme. Cancer Epidemiol 2014; 38: 722-727.

20. Nielsen SF, Nordestgaard BG, Bojesen SE Statin use and reduced cancer related mortality. $N$ Engl $J$ Med 2012; 367: 1792-1802.

21. Gazzerro P, Proto MC, Gangemi G, Malfitano AM, Ciaglia $E$, Pisanti $S$, Santoro A, Laezza C, Bifulco $M$. Pharmacological actions of statins: a critical appraisal in the management of cancer. Pharmacol Rev 2012; 64: 102-146.

22. Ferris JS, McCoy L, Neugut Al, Wrensch M, Lai R. HMG COA reductase inhibitors NSAIDs and risk of glioma. Int J Cancer 2012; 131: E1031-1037.

23. Kikuchi $T$, Nagata $Y$ and Abe $T$. In vitro and in vivo antiproliferative effects of simvastatin, an HMG-CoA reductase inhibitor, on human glioma cells. J Neurooncol 1997; 34: 233-239.

24. Yanae M, Tsubaki K, Satou T, Itoh T, Imano M, Yamazoe $Y$, Nishida S. Statin-induced apoptosis via the suppression of ERK1/2 and Akt activation by inhibition of the genanylgeranyl-pyrophosphotase biosynthesis in glioblastoma. J Exp Clin Cancer Res 2011; 30: 74.

25. Wu $H$, Jiang $H$, Dunuy $L$, Xiong $L$, Changsheng $Q$, Zhou $D$, Mahmood A, Chop M. Effect of simvastatin on glioma cell proliferation, migration, and apoptosis. Neurosurgery 2009; 65: 1087-1097.

26. Jiang Z, Zheng $X$, Lytle RA, Higashikubo R, Rich KM. Lovastatin induced up-regulation of the BH3-only protein, Bim, and cell death in glioblastoma cells. J Neurochem 2004; 89: 168-178.

Trop J Pharm Res, July 2018; 17(7): 1450 
27. Seliger C, Meier CR, Becker C, Jick SS, Bogdahn U, Hau $P$, Leitzmann MF. Statin use and risk of glioma: population-based case-control analysis. Eur J Epidemiol 2016; 31: 947-952.

28. Bababeygy SR, Polevaya NV, Youssef S, Sun A, Xiong A, Prugpichailers $T$, Veeravagu A, Hou LC, Steinman L, Tse V. HMG-CoA reductase inhibition causes increased necrosis and apoptosis in an in vivo mouse glioblastoma multiforme model. Anticancer Res 2009; 29: 4901-4908.

29. Jones KD, Couldwell WT, Hinton DR, Su Y, He S, Anker L, Law RE. Lovastatin induces growth inhibition and apoptosis in human malignant glioma cells. Biochem Biophys Res Commun 1994; 205: 1681-1687.

30. Yang J, Yang Q, Yu J, Li X, Yu S, Zhang X. SPOCK1 promotes the proliferation, migration and invasion of glioma cells through PI3K/AKT and Wnt/ $\beta$-catenin signaling pathways. Oncol Rep 2016; 35: 3566-3576.

31. Misirkica M, Janjetovica K, Vucicevica L, Tovilovicb G, Ristic B, Vilimanovich U, Harhaji-Trajkovic L, SumaracDumanovic M, Micic D, Bumbasirevic V, Trajkovic V. Inhibition of AMPK-dependent autophagy enhances in vitro antiglioma effect of simvastatin. Pharmacol Res 2012; 65: 111-119.

32. Son SM, Kang S, Choi H, Mook-Jung I. Statins induce insulin-degrading enzyme secretion from astrocytes via an autophagy-based unconventional secretory pathway. Mol Neurodegener 2015; 10: 56.

33. Wagner RJ, Martin KA, Powell RJ, Rzucidlo EM. Lovastatin induces VSMC differentiation through inhibition of Rheb and mTOR. Am J Physiol Cell Physiol 2010; 299: C119-127.

34. Gao S, Zhang ZM, Shen ZL, Gao K, Chang L, Guo Y, Li $Z$, Wang W, Wang AM. Atorvastatin activates autophagy and promotes neurological function recovery after spinal cord injury. Neural Regen Res 2016; 11: 977-982.

35. Wang W, Wang $H$, Geng $Q X$, Wang HT, Miao W, Cheng $B$, Zhao D, Song GM, Leanne G, Zhao Z. Augmentation of autophagy by atorvastatin via Akt/mTOR pathway in spontaneously hypertensive rats. Hypertens Res 2015; 38: 813-820.

36. Davis RJ. Signal transduction by the JNK group of MAP kinases. Cell 2000; 103: 239-252.

37. Bae MA, Song BJ. Critical role of $c$-jun N-terminal protein kinase activation in troglitazone-induced apoptosis of human HepG2 hepatoma cells. Mol Pharmacol 2003; 63: 401-408.

38. Koyuturk M, Ersoz M, Altiok N. Simvastatin induces proliferation inhibition and apoptosis in C6 glioma cells via c-jun N-terminal kinase. Neurosci Lett 2004; 370: 212-217.

39. Ohba $S$, Hirose $Y$, Kawase $T$, Sano $H$. Inhibition of C-Jun $N$-terminal kinase enhances temozolomide-induced cytotoxicity in human glioma cells. J Neurooncol 2009; 95: 307-316.

40. Kimberley FC, Screaton GR. Following a TRAIL: update on a ligand and its five receptors. Cell Res 2004; 14: 359-372.
41. Wiley SR, Schooley K, Smolak PJ, Din WS, Huang CP, Nicholl JK, Sutherland GR, Smith TD, Rauch C, Smith $C A$, Goodwin RG. Identification and characterization of a new member of the TNF family that induces apoptosis. Immunity 1995; 3: 673-682.

42. Chan DY, Chen CG, Poon WS, Liu PC. Lovastatin sensitized human glioblastoma cells to TRAIL-induced apoptosis. J Neurooncol 2008; 86: 273-283.

43. Jin Z, Dicker DT, EI Deiry WS. Enhanced sensitivity of G1 arrested human cancer cells suggests a novel therapeutic strategy using a combination of simvastatin and TRAIL. Cell Cycle 2002; 1: 82-89.

44. Wu J, Wong WW, Khosravi F, Minden MD, Penn LZ. Blocking the Raf/MEK/ERK pathway sensitizes acute myelogenous leukemia cells to lovastatin-induced apoptosis. Cancer Res 2004; 64: 6461-6468.

45. Du W, Pang C, Xue $Y$, Zhang $Q$, Wei $X$. Dihydroartemisinin inhibits the Raf/ERK/MEK and PI3K/AKT pathways in glioma cells. Oncol Lett 2015; 10 : 3266-3270.

46. Roberts PJ, Der CJ. Targeting the Raf-MEK-ERK mitogen-activated protein kinase cascade for the treatment of cancer. Oncogene 2007; 26: 3291-3310.

47. Luo M, Liu Q, He M, Yu Z, Pi R, Li M, Yang X, Wang S, Liu A. Gartanin induces cell cycle arrest and autophagy and suppresses migration involving PI3K/Akt/mTOR and MAPK signaling pathway in human glioma cells. J Cell Mol Med 2017; 21: 46-57.

48. Markovic DS, Vinnakota $K$, Chirasani S, Synowitz M, Raguet $H$, Stock $K$, Sliwa M, Lehmann S, Kälin R, van Rooijen $N$, et al. Gliomas induce and exploit miewdiM MTI-MMP expression for tumor expansion. Proc Natl Acad Sci U S A 2009; 106: 12530-12535.

49. Chandrasekar B, Mummidi S, Mahimainathan L, Patel DN, Bailey SR, Imam, SZ, Greene WC, Valente AJ. Interleukin-18-induced human coronary artery smooth muscle cell migration is dependent on NF-kappaB- and AP-1-mediated matrix metalloproteinase-9 expression and is inhibited by atorvastatin. J Biol Chem 2006; 281 : 15099-15109.

50. Tapia-P6rez JH, Kirehes E, Mawrin C, Firsching $R$, Schneider T. Cytotoxic effect of different statins and thiazolidinediones on malignant dioma cells. Cancer Chemother Pharmaeol 2011; 67: 1193-1201.

51. Mahajan N, Dhawan V. Inhibition of C-reactive protein induced expression of matrix metalloproteinases by atorvastatin in THP-1 cells. Mol Cell Biochem 2010; 338: 77-86.

52. Fromigue $O$, Hamidouche Z, Marie PJ. Blockade of the RhoA-JNK-C-Jun-MMP2 cascade by atorvastatin reduces osteosarcoma cell invasion. J Biol Chem 2008; 283: 30549-30556.

53. Yongjun $Y$, Shuyun $H$, Lei $C$, Xiangrong $C$, Zhilin $Y$, Yiquan K. Atorvastatin suppresses glioma invasion and migration by reducing microglial MT1-MMP expression. J Neuroimmunol 2013; 260: 1-8. 